\title{
Relationship between Serum 25(OH)D and Depression: Causal Evidence from a Bi-Directional Mendelian Randomization Study
}

\author{
Anwar Mulugeta ${ }^{1,2,3,4}(\mathbb{D}$, Amanda Lumsden $1,3,4(\mathbb{D}$ and Elina Hyppönen $1,3,4,5 *$ \\ 1 Australian Centre for Precision Health, University of South Australia Cancer Research Institute, University of \\ South Australia, Adelaide, SA 5000, Australia; anwarmulugeta.gebremichael@unisa.edu.au (A.M.); \\ amanda.lumsden@unisa.edu.au (A.L.) \\ 2 Department of Pharmacology and Clinical Pharmacy, College of Health Sciences, Addis Ababa University, \\ Addis Ababa 1000, Ethiopia \\ 3 South Australian Health and Medical Research Institute, Adelaide, SA 5000, Australia \\ 4 Unit of Clinical and Health Sciences, University of South Australia, Adelaide, SA 5000, Australia \\ 5 Population, Policy and Practice, UCL Great Ormond Street Institute of Child Health, London WC1N 1EH, UK \\ * Correspondence: elina.hypponen@unisa.edu.au; Tel.: +61-(08)-83022518
}

Citation: Mulugeta, A.; Lumsden, A.; Hyppönen, E. Relationship between Serum 25(OH)D and Depression: Causal Evidence from a Bi-Directional Mendelian Randomization Study. Nutrients 2021, 13, 109.

https://doi.org/10.3390/nu13010109

Received: 10 November 2020 Accepted: 25 December 2020 Published: 30 December 2020

Publisher's Note: MDPI stays neutral with regard to jurisdictional clai$\mathrm{ms}$ in published maps and institutional affiliations.

Copyright: (C) 2020 by the authors. Licensee MDPI, Basel, Switzerland. This article is an open access article distributed under the terms and conditions of the Creative Commons Attribution (CC BY) license (https:// creativecommons.org/licenses/by/ $4.0 /)$.

\begin{abstract}
The relationship between depression and vitamin D deficiency is complex, with evidence mostly from studies affected by confounding and reverse causality. We examined the causality and direction of the relationship between 25-hydroxyvitamin $\mathrm{D}(25(\mathrm{OH}) \mathrm{D})$ and depression in bidirectional Mendelian randomization (MR) analyses using information from up to 307,618 white British participants from the UK Biobank and summary results from the SUNLIGHT $(n=79,366)$ and Psychiatric Genomics consortia (PGC 113,154 cases and 218,523 controls). In observational analysis, the odds of depression decreased with higher 25(OH)D concentrations (adjusted odds ratio (OR) per $50 \%$ increase $0.95,95 \%$ CI 0.94-0.96). In MR inverse variance weighted (IVW) using the UK Biobank, there was no association between genetically determined serum $25(\mathrm{OH}) \mathrm{D}$ and depression (OR per $50 \%$ higher $0.97,95 \% \mathrm{CI} 0.90-1.05$ ) with consistent null association across all MR approaches and in data from PGC consortium. In contrast, genetic liability to depression was associated with lower $25(\mathrm{OH}) \mathrm{D}$ concentrations (MR IVW $-3.26 \%,-4.94 \%--1.55 \%$ ), with the estimates remaining generally consistent after meta-analysing with the consortia. In conclusion, we found genetic evidence for a causal effect of depression on lower $25(\mathrm{OH}) \mathrm{D}$ concentrations, however we could not confirm a beneficial effect of nutritional vitamin $\mathrm{D}$ status on depression risk.
\end{abstract}

Keywords: 25(OH)D; nutritional vitamin D status; depression; observational analysis; Mendelian randomization; UK Biobank

\section{Introduction}

Studies suggest a link between hypovitaminosis $\mathrm{D}$ and a diverse range of conditions, including an increased risk of depression [1,2]. Vitamin D receptors (VDR) and the enzymes required for local vitamin $\mathrm{D}$ activation are expressed in the brain [3] and there are plausible biological mechanisms that could mediate an effect of vitamin D deficiency to depression. For example, hormonal vitamin D promotes the production of neurotransmitters including serotonin, dopamine and norepinephrine, through VDR-mediated transcriptional upregulation of hydroxylase genes that catalyse their synthesis [4,5]. Decreased levels of these neurotransmitters are associated with depression, and drugs that promote their availability represent effective antidepressant treatments. Active vitamin $\mathrm{D}$ also has anti-inflammatory properties, which may counter the increase in inflammatory cytokines associated with depression [1]. However, results from randomized control trials (RCT) investigating potential benefits of vitamin $\mathrm{D}$ supplementation in depression have been inconsistent. A recent large-scale RCT [6] and an earlier systematic review and meta-analysis including nine 
RCTs $(n=4923)$, investigated the effects of vitamin D supplementation in reducing depressive symptoms in adults, but found no effects [7]. In contrast, another review comparing 15 RCTs, and assessing the studies based on their methodological merit, found that vitamin D supplementation produced favourable results in six of seven studies 'without biological flaws', but only in three of the eight studies 'with flaws'; in which the 'flaws' were defined as lack of $25(\mathrm{OH}) \mathrm{D}$ measures or reduced $25(\mathrm{OH})$ levels in the intervention group, and those with baseline $25(\mathrm{OH})$ level indicated sufficiency [8]. Beneficial effects of vitamin D supplementation on depression have been observed when used as adjunct treatment with antidepressants [9], and amongst patients with psychiatric disorders [10]. While the benefits of vitamin $\mathrm{D}$ in the prevention of depression remain uncertain, it is also possible that the observational association between $25(\mathrm{OH}) \mathrm{D}$ and depression can be due to links between behaviour and vitamin D-related covariates. Indeed, many of the characteristics and behaviours that associate with depression, such as obesity, lower use of vitamin supplements, poor-quality diet, and less time spent outdoors, can also limit vitamin D intakes from the diet and/or sunlight-induced synthesis in the skin [11].

Currently, it is uncertain whether the relationship between $25(\mathrm{OH}) \mathrm{D}$ concentrations and depression is causal, and whether it operates in one or both directions. Mendelian randomization (MR) is a genetic approach that allows for the testing of causality even in situations where clinical trials may be difficult to conduct. As it approximates the exposure using genetic variants that are determined at the time of conception (which do not change in response to health or lifestyles), it allows us to overcome common problems of observational studies including reverse causality and confounding (Figure 1). Prior MR studies have been either unidirectional (i.e., 25(OH)D to depression) or based on information only from GWAS summary data [12-14]. In this study, we use information from up to 307,618 participants from the UK biobank together with summary level data from consortia meta-analyses to investigate the causality and direction of the association between $25(\mathrm{OH}) \mathrm{D}$ and depression. If lower $25(\mathrm{OH}) \mathrm{D}$ has a causal effect on depression, genetically instrumented lower $25(\mathrm{OH}) \mathrm{D}$ concentration should be associated with a proportionately greater risk of depression. In the other direction, if depression leads to low vitamin $\mathrm{D}$ status, genetic variants associated with depression risk should associate with lower $25(\mathrm{OH}) \mathrm{D}$ concentrations.

Panel A

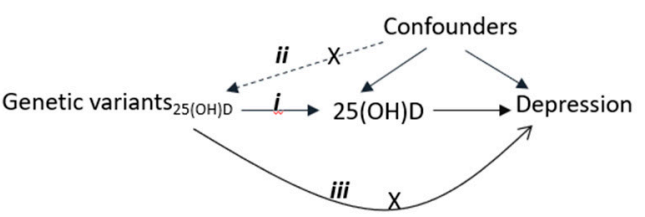

Panel B

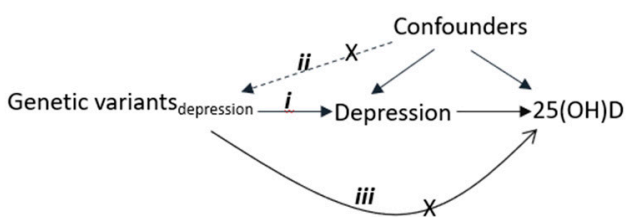

Figure 1. Diagram showing the principle of Mendelian randomization (MR) analysis [15]. Panel A shows the association between 25(OH) and depression, and Panel B between depression and 25(OH)D. The numbers reflect the MR assumptions that the genetic variants should (i) be associated with the exposure, (ii) not associated with the confounders of exposureoutcome association, and (iii) not affect the outcome through pathways other than exposure.

\section{Methods}

\subsection{Participants}

UK Biobank is a large prospective cohort of over 500,000 individuals aged 37-73 years during recruitment in one of the 22 assessment centres between 2006 and 2010 [16]. The cohort contains an extensive range of genetic and phenotypic data including information on lifestyle factors, diseases, various physical measures, and blood and urine biomarkers. A detailed description of the cohort can be found elsewhere [16,17]. The analysis for this study was restricted to unrelated white British ancestry participants (as evidenced by self-report and genetic analysis [17]) for whom complete genetic information is available ( $n=337,484$; Figure S1). For MR analysis investigating effects of $25(\mathrm{OH}) \mathrm{D}$ on depression, 
we included 251,962 participants with complete information on depression. We excluded participants $(n=85,522)$ for whom depression status was unclear, including participants who reported less than two weeks duration of depression/unenthusiasm $(n=429)$ and those who lacked this information $(n=85,093)$ [18]. Participants with complete information on $25(\mathrm{OH}) \mathrm{D}(n=307,618)$ were used for the MR analysis of the effect of depression on 25(OH)D. For observational analyses, data were used from 229,832 participants for whom both $25(\mathrm{OH}) \mathrm{D}$ and depression information was available. We also used summary data from the SUNLIGHT consortium $(n=79,366)$ [19] and Psychiatric Genetic Consortium $(113,154$ cases and 218,523 controls), which together with the data from UK Biobank allowed us to conduct MR analyses for the effect of 25(OH)D on depression in up to 424,967 participants, and analyses of depression on $25(\mathrm{OH}) \mathrm{D}$ in up to 386,984 participants.

UK Biobank obtained informed consent from each participant, and ethical approval was granted by the National Information Governance Board for Health and Social Care and North West Multicentre Research Ethics Committee [20]. The current study is approved by the UK Biobank under application number 10171.

Depression was defined using information from touchscreen questionnaires, nurse-led interviews, and linked hospital registry records. Participants who had seen a general practitioner or a psychiatrist for anxiety, tension, nervousness or depression, and reported depression or unenthusiasm of at least two weeks duration were recoded as having depression [18]. Additional cases were identified from hospital diagnoses (ICD-10 F32 or F33 or the corresponding ICD-9 codes) obtained from Hospital Episode Statistics (HES). Individuals in the control group were those who had not seen a general practitioner or psychiatrist for anxiety, tension, nervousness or depression, and who had no hospital diagnosed depression, and no self-reported depression.

$25(\mathrm{OH}) \mathrm{D}$ concentrations were measured from blood samples taken at baseline assessment with details on sample storage, processing, analysis and related quality controls reported elsewhere $[21,22]$. As covariates in the observational analyses, we included information on age, sex, assessment centre, date of blood sample collection, socioeconomic and lifestyle variables to account for potential confounding. Covariates were based on self-reported data from the baseline assessment, with the exception of the Townsend index reflecting area deprivation, which was derived from participants' post codes as recorded in the National Health System primary care trust registries [23]. Education was based on highest qualification and grouped as "none", "A-levels and below" and "degree or professional". Based on the employment status and working hours, we grouped employment in to six categories as "lowest (first quartile) working hour group", "second quartile", "third quartile" and "highest (fourth quartile) working hour", "retired" and "not working" groups. Body mass index (BMI) was calculated as weight $(\mathrm{kg}) / \mathrm{height}^{2}\left(\mathrm{~m}^{2}\right)$ and categorized based on World Health Organization recommendation [24] as "underweight" $\left(<18.5 \mathrm{~kg} / \mathrm{m}^{2}\right)$, "normal" $\left(\geq 18.5 \mathrm{~kg} / \mathrm{m}^{2}\right.$ and $\left.<25 \mathrm{~kg} / \mathrm{m}^{2}\right)$, "overweight" $\left(\geq 25 \mathrm{~kg} / \mathrm{m}^{2}\right.$ and $\left.<30 \mathrm{~kg} / \mathrm{m}^{2}\right)$ and "obese" $\left(\geq 30 \mathrm{~kg} / \mathrm{m}^{2}\right)$. Physical activity was grouped as "none", "light/moderate", and "strenuous activity"; smoking and alcohol consumption as "never", "previous", and "current"; and long-standing illness, disability or infirmity (hereafter referred as "long standing illness") as "No" and "Yes". Participants were asked about the frequency of oily or non-oily fish, and cheese consumption, that were categorized here as "never", "less than once a week", "once a week", and "more than once a week". Participants were asked about dietary restrictions, which were grouped here as "no egg or dairy containing food", "no wheat containing food", "no sugar, or sugar containing food", and "eat all above". Information regarding time spent outdoors in a typical day of summer and winter ("none", "less than an hour", "one", "two", "three", "four", "five", "at least six hours"), and sun protection use ("never/rarely", "sometimes", "most of the time", "always", and "do not go out in sun"), were included in the covariates. 


\subsection{Genetic Variants of Serum 25(OH)D and Depression}

Genotyping, imputation and related quality control were performed by the UK Biobank genetic team, for which detailed information can be found elsewhere [17]. Genotyping was done using UK BiLEVE $(n \sim 50,000)$ and UK Biobank axiom array $(n \sim 450,000)$, with the two arrays having $95 \%$ similarity in marker contents. Imputation was performed using the Haplotype reference consortium, and UK10K and 1000 genome reference panels. We used the third release UK Biobank genetic imputation dataset for extracting the genetic variants of interest.

We used six variants associated with serum level of $25(\mathrm{OH}) \mathrm{D}$ that attained genomewide significance in a recent genome wide association study (GWAS), with these variants explaining $\sim 3 \%$ of the variability in serum level of $25(\mathrm{OH}) \mathrm{D}$ [20]. In UK Biobank participants, these variants were in Hardy-Weinberg equilibrium $(p>0.17)$ and had minor allele frequency (MAF) of at least 0.18 , and good imputation quality (INFO score $\geq 0.96$ ) (Table S1). In a sensitivity analysis, we used 122 variants (excluding insertion and deletion type of genetic variants) associated with $25(\mathrm{OH}) \mathrm{D}$ that are identified in a recent GWAS that included UK Biobank [25].

We used 44 variants from a recent meta-analysis of GWAS on major depressive disorder (MDD) to instrument depression [26]. As the UK Biobank contributed to $10 \%$ of the cases and $4 \%$ of the controls in this GWAS meta-analyses, to minimize potential bias from sample overlap [27], we conducted sensitivity analyses using 17 MDD-related variants identified in an earlier MDD GWAS, that did not include UK Biobank participants [28]. All MDD-related variants were in Hardy-Weinberg equilibrium $(p>0.0003)$, with minor allele frequency greater than 0.07, and INFO score greater than 0.95 in the UK Biobank (Table S2 and Figure S2).

\subsection{Statistical Analyses}

We included observational and MR analysis approaches (Supplementary note and Figure S3) to investigate the bi-directional association between $25(\mathrm{OH}) \mathrm{D}$ and depression. Natural log-transformed 25(OH)D concentrations were used for analyses involving $25(\mathrm{OH}) \mathrm{D}$ as a continuous variable. In the model with $25(\mathrm{OH}) \mathrm{D}$ as the predictor, a converting factor of (1.50) ${ }^{\log O R}$ was used to reflect the OR of depression per $50 \%$ change in $25(\mathrm{OH}) \mathrm{D}$. In the model including $25(\mathrm{OH}) \mathrm{D}$ as the outcome, a conversion factor of $100 \times(\exp ($ beta $)-1)$ enabled the effect estimate to be interpreted as percentage change (increase or decrease) in $25(\mathrm{OH}) \mathrm{D}$ by depression. Analyses were carried out using Stata v. 16.0 (StataCorp LLC, College Station, TX, USA) and R v. 3.5.0 (R Foundation for Statistical Computing, Vienna, Austria).

In the observational analysis, we tested whether the association between $25(\mathrm{OH}) \mathrm{D}$ and depression was different among males and females, and in different age groups, using related interaction terms. Where interactions were found, results are presented by sex and age stratification. We explored the association between serum $25(\mathrm{OH}) \mathrm{D}$ and depression using logistic regression with adjustment made for ranges of covariates in three models. The first model adjusted for basic covariates (age, sex, assessment centre, and blood sample collection date). The second model adjusted for basic plus socioeconomic-related covariates (Townsend deprivation, education, and employment). A final model additionally adjusted for lifestyle factors including smoking, alcohol consumption, BMI, physical activity, sun exposure in summer and winter, use of sun protection, diet restriction, fish and cheese consumption and long-standing illness. Using the same model structures, we investigated the association between depression and $25(\mathrm{OH}) \mathrm{D}$. We conducted sensitivity analyses excluding serum $25(\mathrm{OH}) \mathrm{D}$ levels from aliquots affected by sample dilution bias $(n=7041)$ [22] $25(\mathrm{OH}) \mathrm{D}$ levels were categorized into four groups defined as: $<25 \mathrm{nmol} / \mathrm{L}, \geq 25$ and $<50$, $\geq 50$ and $<75$ and $>75 \mathrm{nmol} / \mathrm{L}$ for analysis involving $25(\mathrm{OH}) \mathrm{D}$ as a categorical indicator.

To provide genetic causal evidence of the $25(\mathrm{OH}) \mathrm{D}$-depression bi-directional association, we used two-sample MR approaches using variant-exposure estimates from primary GWAS on 25(OH)D [20] and depression [26]. Variant-outcome estimates were derived both 
from the UK Biobank and from consortia meta-analyses [26], with estimates meta-analysed where relevant. The primary analyses used inverse variance weighted MR (MR IVW). As this approach assumes that there is no horizontal pleiotropy, i.e., that all the SNPs included are valid instruments, we conducted sensitivity analyses using four additional MR approaches, that allow one or more genetic variants to have horizontal pleiotropic effects, but which are relatively more power demanding. These include weighted median [29], weighted mode [30], MR-Egger [31], and MR-PRESSO [32], with each relying on different assumptions. The intercept from MR-Egger indicates the extent of directional pleiotropy [31]. Additional tests for pleiotropy and its impact on MR estimates were further explored using MR-PRESSO. MR-PRESSO involves three tests: MR-PRESSO global test, which detects the presence of unbalanced net-horizontal pleiotropy; MR-PRESSO outlier test, which identifies specific horizontal pleiotropic outlying variants; and MR-PRESSO distortion test, which assesses the change in the causal estimate after removal of pleiotropic outlying variants [32]. We also included leave-one-out analysis to examine whether the associations examined were sensitive to the effects by individual variants. In a sensitivity analyses involving 122 variants associated with 25(OH)D from recent GWAS [25], we collected the variant-25(OH)D association estimates from UK Biobank participants with no depression (from control) and the variant-depression association estimates from all UK biobank individuals to minimize the bias in the MR estimates from the sample overlap [27].

\section{Results}

Of the 229,832 individuals included in the observational analysis, $49.2 \%$ were females (Table 1). Generally, across the covariates, categories associated with lower 25(OH)D concentrations were also reflective of a higher prevalence of depression (Table 1 and Table S3). Individuals who were obese, not physically active, never consumed oily fish, had longstanding illness, or those who do not go in sunshine had lower $25(\mathrm{OH}) \mathrm{D}$ concentrations and higher prevalence of depression compared to the others.

Table 1. Prevalence of depression and summary of serum 25(OH)D (in nanomoles per litre, nmol/L) across different characteristics.

\begin{tabular}{|c|c|c|c|c|c|}
\hline & \multirow{2}{*}{$n(\%)$} & \multicolumn{2}{|c|}{ Depression } & \multicolumn{2}{|c|}{ Serum $25(\mathrm{OH}) \mathrm{D}$ in $\mathrm{nmol} / \mathrm{L}$} \\
\hline & & $n(\%)$ & $p$-Value ${ }^{1}$ & Median (IQR) & $p$-Value ${ }^{2}$ \\
\hline Sex & & & $<1.0 \times 10^{-300}$ & & 0.02 \\
\hline Male & $116,698(50.8)$ & $11,292(9.7)$ & & $48.8(34.4,64.0)$ & \\
\hline Female & $113,134(49.2)$ & $18,855(16.7)$ & & $48.9(34.3,63.9)$ & \\
\hline Age & & & $7.7 \times 10^{-87}$ & & $<1.0 \times 10^{-300}$ \\
\hline $39-49$ years & $51,191(22.3)$ & $7201(14.1)$ & & $46.1(32.1,62.0)$ & \\
\hline $50-59$ years & $73,641(32.0)$ & $10,548(14.3)$ & & $47.5(33.2,62.8)$ & \\
\hline $60-73$ years & $105,000(45.7)$ & $12,398(11.8)$ & & $51.0(36.6,65.4)$ & \\
\hline BMI & & & $4.5 \times 10^{-154}$ & & $<1.0 \times 10^{-300}$ \\
\hline Underweight, $<18.5 \mathrm{~kg} / \mathrm{m}^{2}$ & $1083(0.5)$ & $167(15.4)$ & & $48.1(31.2,67.2)$ & \\
\hline Normal, $(\geq 18.5$ and $<25) \mathrm{kg} / \mathrm{m}^{2}$ & $75,087(32.7)$ & $9031(12.0)$ & & $52.3(36.7,67.6)$ & \\
\hline Overweight, $(\geq 25$ and $<30) \mathrm{kg} / \mathrm{m}^{2}$ & $98,778(43.0)$ & $11,961(12.1)$ & & $49.8(35.6,64.3)$ & \\
\hline Obese, $\geq 30 \mathrm{~kg} / \mathrm{m}^{2}$ & $54,151(23.6)$ & $8821(16.3)$ & & $42.9(30.2,57.2)$ & \\
\hline Missing & $733(0.3)$ & $167(22.8)$ & & $39.7(25.9,56.0)$ & \\
\hline Education & & & $3.4 \times 10^{-13}$ & & $6.0 \times 10^{-66}$ \\
\hline None & $38,458(16.7)$ & $5030(13.1)$ & & $49.7(34.8,65.0)$ & \\
\hline NVQ/CSE/A levels & $81,147(35.3)$ & $11,044(13.6)$ & & $49.7(35.0,64.9)$ & \\
\hline Degree/professional & $108,287(47.1)$ & $13,881(12.8)$ & & $47.9(33.8,62.8)$ & \\
\hline Missing & $1940(0.8)$ & $192(9.9)$ & & $50.0(34.7,65.0)$ & \\
\hline Physical activity & & & $2.0 \times 10^{-200}$ & & $<1.0 \times 10^{-300}$ \\
\hline None & $12,000(5.2)$ & 2542 (21.2) & & $35.4(23.9,51.7)$ & \\
\hline Light/moderate & $191,144(83.2)$ & $25,075(13.1)$ & & $48.9(34.7,63.7)$ & \\
\hline Strenuous sports & $25,932(11.3)$ & $2337(9.0)$ & & $54.2(39.3,69.2)$ & \\
\hline Missing & $756(0.3)$ & $193(25.5)$ & & $38.6(24.7,56.1)$ & \\
\hline Oily fish consumption & & & $4.8 \times 10^{-36}$ & & $<1.0 \times 10^{-300}$ \\
\hline Never & $24,100(10.5)$ & 3775 (15.7) & & $44.2(29.7,60.6)$ & \\
\hline$<$ Once a week & $76,738(33.4)$ & $10,090(13.2)$ & & $47.1(32.6,62.6)$ & \\
\hline Once a week & $87,870(38.2)$ & $10,737(12.2)$ & & $49.9(35.7,64.6)$ & \\
\hline >Once a week & $40,130(17.5)$ & $5400(13.5)$ & & $52.2(38.2,66.4)$ & \\
\hline
\end{tabular}


Table 1. Cont.

\begin{tabular}{|c|c|c|c|c|c|}
\hline & \multirow{2}{*}{$n(\%)$} & \multicolumn{2}{|c|}{ Depression } & \multicolumn{2}{|c|}{ Serum $25(\mathrm{OH}) \mathrm{D}$ in $\mathrm{nmol} / \mathrm{L}$} \\
\hline & & $n(\%)$ & $p$-Value ${ }^{1}$ & Median (IQR) & $p$-Value ${ }^{2}$ \\
\hline Missing & $994(0.4)$ & 145 (14.6) & & $45.9(30.6,61.9)$ & \\
\hline Sun protection use & & & $1.8 \times 10^{-60}$ & & $<1.0 \times 10^{-300}$ \\
\hline Do not go in sunshine & $1048(0.5)$ & $251(24.0)$ & & $31.7(21.1,45.7)$ & \\
\hline Never/rarely & $18,794(8.2)$ & $2506(13.3)$ & & $43.9(29.8,59.8)$ & \\
\hline Sometimes & $77,559(33.7)$ & $9443(12.2)$ & & $48.6(34.3,63.5)$ & \\
\hline Most of the time & $84,409(36.7)$ & $11,164(13.2)$ & & $49.7(35.4,64.6)$ & \\
\hline Always & $47,902(20.8)$ & $6764(14.1)$ & & $49.7(34.9,65.0)$ & \\
\hline Missing & $120(0.1)$ & $19(15.8)$ & & $33.2(22.4,48.6)$ & \\
\hline Long standing illness & & & $<1.0 \times 10^{-300}$ & & $5.9 \times 10^{-280}$ \\
\hline No & $156,658(68.2)$ & $15,275(9.8)$ & & $49.7(35.3,64.5)$ & \\
\hline Yes & $68,590(29.8)$ & $14,113(20.6)$ & & $46.8(32.2,62.6)$ & \\
\hline Missing & $4584(2.0)$ & $759(16.6)$ & & $46.9(32.9,61.9)$ & \\
\hline
\end{tabular}

${ }^{1} p$-value from likelihood ratio test in logistic regression model adjusted for adjusted for sex, age, assessment centre, and date of blood sample collected. ${ }^{2} p$-value from likelihood ratio test in linear regression model adjusted for sex, age, assessment centre, and date of blood sample collected. See Table S3 for more list of covariates.

The odds of depression were lower in individuals with higher compared to lower $25(\mathrm{OH}) \mathrm{D}$ and the association remained after adjusting for socioeconomic and lifestyle covariates (Table 2). Despite some evidence of interaction between 25(OH)D and sex on their association with depression $\left(P_{\text {sex-interaction }}=0.004\right)$, we saw evidence for a protective association both among males and females, with a slightly stronger association in males (Table S4). We did not find evidence for an interaction between 25(OH)D and age on depression $\left(P_{\text {age-interaction }}=0.07\right)$. Sensitivity analyses excluding serum $25(\mathrm{OH}) \mathrm{D}$ data affected by sample dilution bias provided consistent results with the analyses including all aliquots (Table S5).

Table 2. Association between serum 25(OH)D and depression.

\begin{tabular}{|c|c|c|c|c|c|}
\hline & \multirow[b]{2}{*}{$n(\%)$} & \multirow[b]{2}{*}{ Depression $n(\%)$} & \multicolumn{3}{|c|}{ Odds of Depression $(n=202,413)^{6}$} \\
\hline & & & $\begin{array}{l}\text { Basic }^{1} \text { OR } \\
(95 \% \mathrm{CI})\end{array}$ & $\begin{array}{l}\text { Socioeconomic }^{2} \\
\text { OR }(95 \% \mathrm{CI})\end{array}$ & $\begin{array}{c}\text { Lifestyle }^{3} \text { OR } \\
(95 \% \mathrm{CI})\end{array}$ \\
\hline \multicolumn{6}{|l|}{ Serum 25(OH)D level ${ }^{4}$} \\
\hline$<25$ & $21,688(10.7)$ & 3209 (14.8) & Reference & Reference & Reference \\
\hline$\geq 25$ and $<50$ & $82,389(40.7)$ & $10,548(12.8)$ & $0.72(0.68,0.75)$ & $0.76(0.73,0.80)$ & $0.85(0.81,0.89)$ \\
\hline$\geq 50$ and $<75$ & $72,843(36.0)$ & $9206(12.6)$ & $0.64(0.61,0.67)$ & $0.70(0.66,0.73)$ & $0.83(0.79,0.87)$ \\
\hline$>75$ & $25,493(12.6)$ & $3312(13.0)$ & $0.62(0.58,0.66)$ & $0.67(0.63,0.71)$ & $0.83(0.78,0.88)$ \\
\hline Per $50 \%$ higher serum $25(\mathrm{OH}) \mathrm{D}^{5}$ & 202,413 & $26,270(13.0)$ & $0.87(0.86,0.89)$ & $0.90(0.88,0.91)$ & $0.95(0.94,0.96)$ \\
\hline$P_{\text {trend }}$ & & & $2.1 \times 10^{-72}$ & $4.9 \times 10^{-50}$ & $4.0 \times 10^{-12}$ \\
\hline$P_{\text {curvature }}$ & & & $8.0 \times 10^{-12}$ & $2.4 \times 10^{-6}$ & $2.8 \times 10^{-4}$ \\
\hline $\mathrm{P}_{\text {sex-interaction }}$ & & & $3.8 \times 10^{-6}$ & $7.9 \times 10^{-4}$ & $9.4 \times 10^{-4}$ \\
\hline$P_{\text {age-interaction }}$ & & & 0.03 & 0.04 & 0.07 \\
\hline
\end{tabular}

1 Basic model included adjustment for basic covariates including age, sex, assessment centre, and date of blood sample collected. 2 Socioeconomic model included adjustment for basic and socioeconomic-related covariates including education, Townsend deprivation index, and employment. ${ }^{3}$ Lifestyle model included adjustment for basic, socioeconomic and lifestyle-related covariates including smoking, alcohol consumption, BMI, physical activity, fish and cheese consumptions, dietary restriction, sun exposure [in summer or winter], use of sun protection and long-standing illness. ${ }^{4}$ Serum $25(\mathrm{OH}) \mathrm{D}$ level expressed in nanomoles per litres (nmol/L) unit. ${ }^{5}$ Natural log-transformed $25(\mathrm{OH}) \mathrm{D}$, and effect estimates transformed to reflect per $50 \%$ higher in $25(\mathrm{OH}) \mathrm{D}$. ${ }^{6}$ Number of individuals in the complete case analyses.

We used six genetic variants from recent $25(\mathrm{OH}) \mathrm{D}$ GWAS in which the genetic risk score explained $2.7 \%$ of the variability in $25(\mathrm{OH}) \mathrm{D}$ in the UK Biobank (Figure S4). The results from two-sample MR analyses did not suggest a causal effect of $25(\mathrm{OH}) \mathrm{D}$ on depression (Figure 2). From MR IVW, the odds of depression per 50\% higher genetically determined serum $25(\mathrm{OH}) \mathrm{D}$ were 0.97 , with similar estimates from analyses using consortia, and estimates after meta-analyses; in all cases the confidence intervals crossed the null but also included the point estimate from observational analyses. The estimates from MR-PRESSO, weighted median, weighted mode and MR-Egger were consistent with the MR IVW estimate and CIs across all methods crossed the null (Figure 2). No evidence of 
directional pleiotropy was identified from MR-Egger intercept, MR-PRESSO outlier and leave-one-out analyses (Figure S5). We carried out further sensitivity analyses separating vitamin D according to those affecting $25(\mathrm{OH}) \mathrm{D}$ synthesis (DHCR7, and CYP2R1) and others, and again found no evidence for a causal association (Table S6). Extended analyses using $12225(\mathrm{OH}) \mathrm{D}$-related variants from recent GWAS [25] found no evidence of causal association between $25(\mathrm{OH}) \mathrm{D}$ and depression (Figure $\mathrm{S6}$ ). Our study was powered $(80 \%$, alpha 0.05 ) to identify up to $8 \%$ lower odds of depression per $50 \%$ higher $25(\mathrm{OH}) \mathrm{D}$.

OR $(95 \% \mathrm{Cl})$

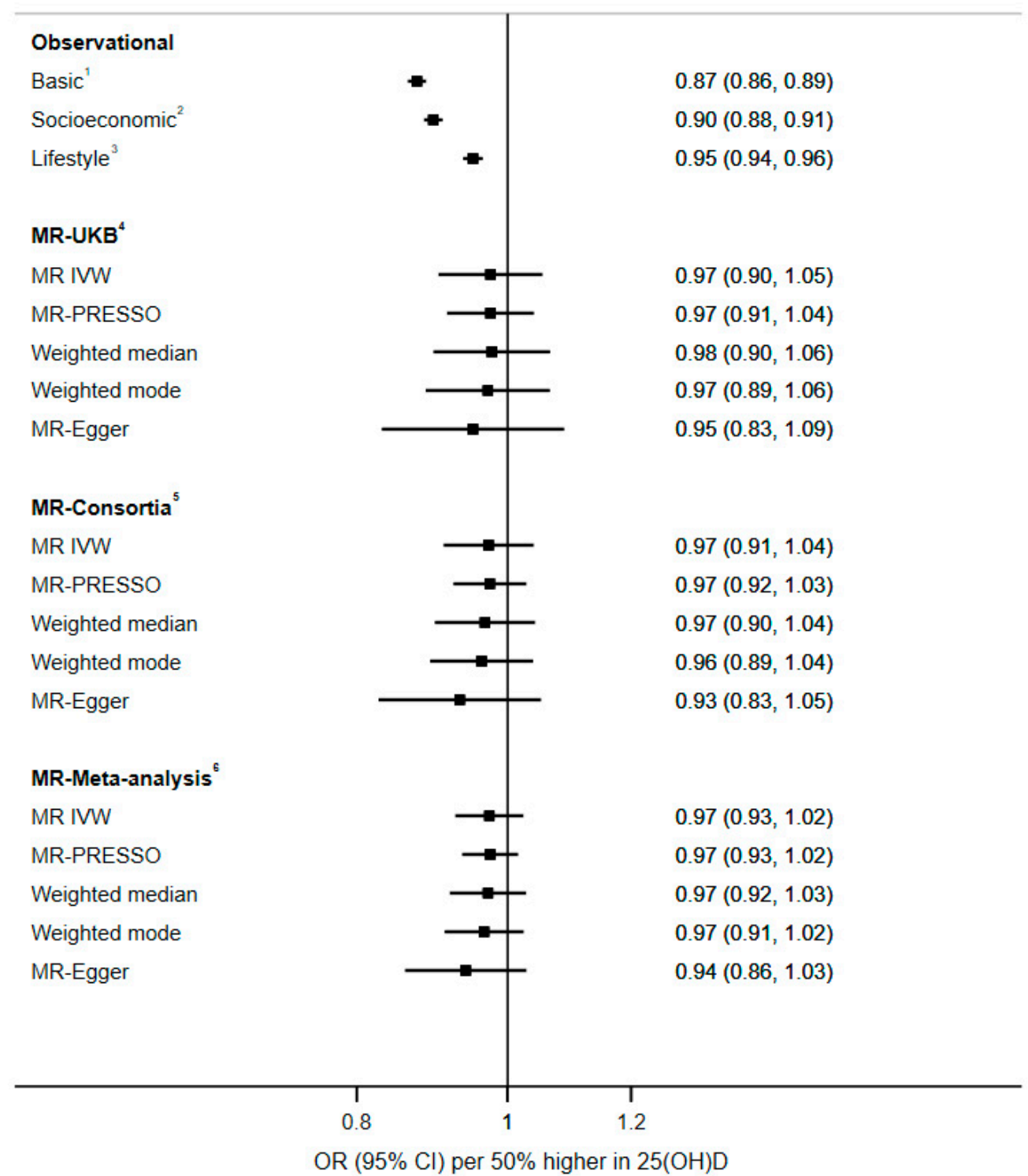

Figure 2. Observational and MR analyses on the association between 25(OH)D and the odds of depression. ${ }^{1}$ Basic model included adjustment for basic covariates including age, sex, assessment centre and date of blood sample collected. ${ }^{2}$ Socioeconomic model included adjustment for basic and socioeconomic-related covariates including education, Townsend deprivation index and employment. ${ }^{3}$ Lifestyle model included adjustment for basic, socioeconomic and lifestyle-related covariates including smoking, alcohol consumption, BMI, physical activity, fish and cheese consumptions, dietary restriction, sun exposure [in summer or winter], use of sun protection, and long-standing illness. ${ }^{4}$ MR analysis based on variantdepression association estimates from UK Biobank. ${ }^{5}$ MR-analysis based on variant-depression association estimates from Wray et al. GWAS. ${ }^{6}$ Meta-analysis of MR estimates from UK Biobank and Wray et al. GWAS. For all MR analysis, variant-25(OH)D estimates were from Jiang et al. GWAS. MR-Egger P-intercept (for all), $p<0.67$. 
The observational analyses indicated that depression was associated with lower $25(\mathrm{OH}) \mathrm{D}$ concentrations $(-1.96 \%, 95 \% \mathrm{CI}-2.50$ to -1.42 ; Figure 3). In MR IVW using $44 \mathrm{MDD}$-related variants from Wray et al. GWAS (Figure S4), serum $25(\mathrm{OH}) \mathrm{D}$ concentrations were $3.26 \%$ lower among individuals with genetically instrumented depression compared to others (Figure 3). Consistent evidence was found using MR-PRESSO and weighted median methods, while the effect estimate from weighted mode analysis were similar, but confidence intervals crossed the null. Despite the MR-Egger estimate being directionally inconsistent with estimates from other methods, the confidence interval was wide. We saw no evidence for horizontal pleiotropy in MR-Egger, MR-PRESSO outlier and leave-one-out analysis (Figure S7). Sensitivity analyses using 17 MDD-related variants that did not include UK Biobank participants $(0.1 \%$ of the variability in MDD vs. $0.2 \%$ with 44 variants) gave causal estimates that were generally consistent with the primary analyses (Figures S8 and S9).

Percent-change $(95 \% \mathrm{Cl})$

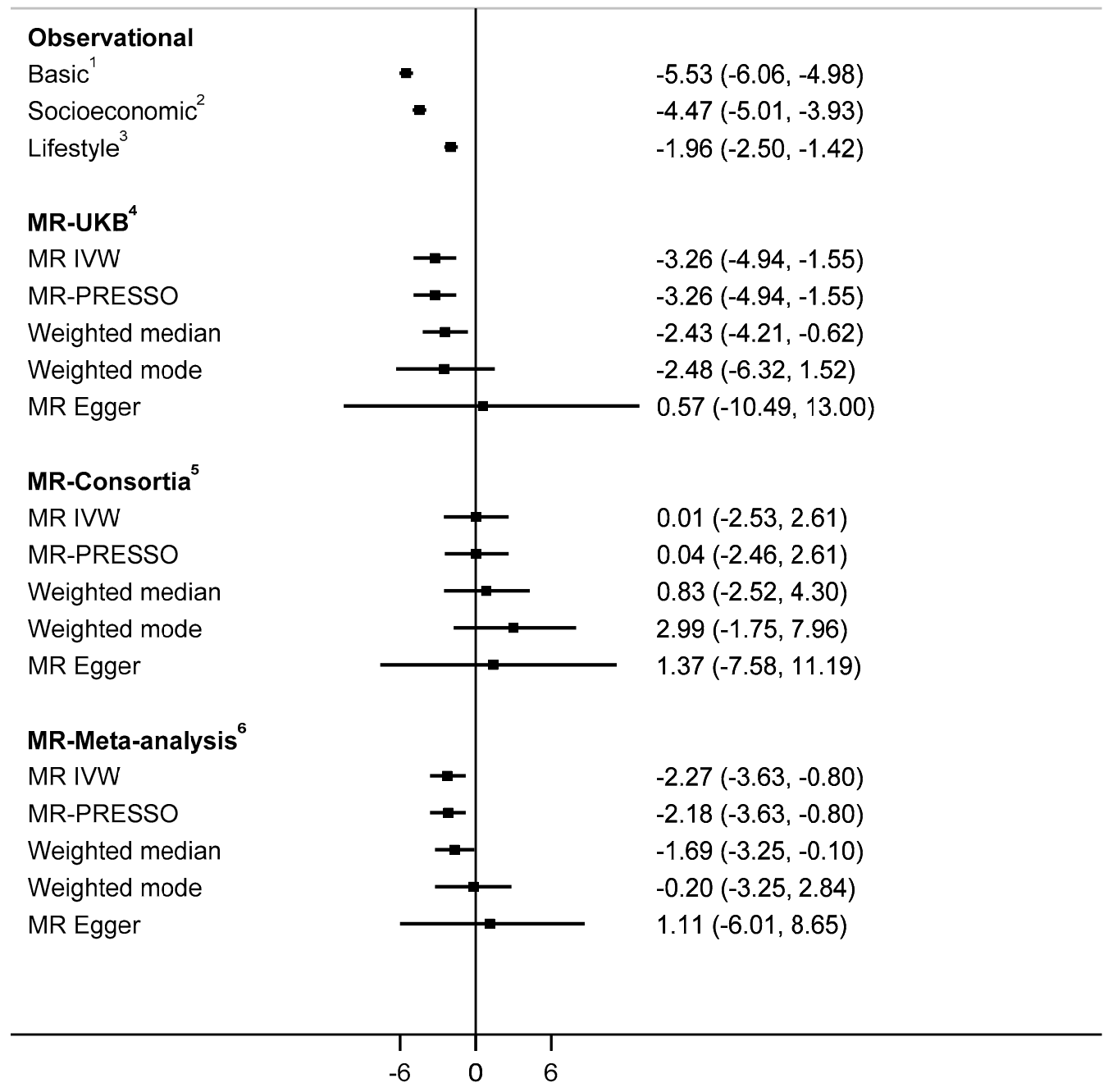

Figure 3. Observational and MR analyses on the association between depression and 25(OH)D concentrations. ${ }^{1}$ Basic model included adjustment for basic covariates including age, sex, assessment centre and date of blood sample collected. ${ }^{2}$ Socioeconomic model included adjustment for basic and socioeconomic-related covariates including education, Townsend deprivation index and employment. ${ }^{3}$ Lifestyle model included adjustment for basic, socioeconomic and lifestyle-related covariates including smoking, alcohol consumption, BMI, physical activity, fish and cheese consumptions, dietary restriction, sun exposure [in summer or winter], use of sun protection and long-standing illness. ${ }^{4} \mathrm{MR}$ analysis based on variant-25(OH) association estimates from UK Biobank. ${ }^{5} \mathrm{MR}$-analysis based on variant-25(OH)D association estimates from Jiang et al. GWAS. ${ }^{6}$ Meta-analysis of MR estimates from UK Biobank and Jiang et al. GWAS. For all MR analysis, variant-depression estimates were from Wray et al. GWAS. MR-Egger P-intercept (for all), $p<0.51$. 


\section{Discussion}

Vitamin D is considered to be an important, modifiable risk factor for several diseases, and low vitamin D status is associated with depression. Despite consistent evidence from observational studies, the direction of the association and whether it is causal has been uncertain. Our study provided no evidence to support a causal role of vitamin D status on the risk of depression. In contrast, we found that depression leads to lower $25(\mathrm{OH}) \mathrm{D}$ concentrations, which may have clinical implications.

Our findings are consistent with those from a recent clinical trial that randomised 18,353 adults to receive vitamin D supplementation or placebo, which reported no differences in the risk of depression during the five years of follow-up [5]. In line with our study, earlier smaller MR studies have also not supported a causal effect of $25(\mathrm{OH}) \mathrm{D}$ on depression [12-14]. The effects of depression on vitamin D status have been less studied, and while an earlier MR study by Milaneschi et al. did not find evidence to support a causal role [12], this may have been due to a lack of power. With the inclusion UK Biobank, and the meta-analysis with information from large-scale consortia, we were able to increase the sample size over four-fold, confirming the causal role of depression and lower $25(\mathrm{OH}) \mathrm{D}$ concentrations.

A true effect of depression on $25(\mathrm{OH}) \mathrm{D}$ concentrations is plausible and may be related to many factors. Depression is often linked to fatigue, isolation and a sedentary lifestyle, which could all translate to more time spent indoors, leading to lower exposure to sunlight. Poor dietary choices, or reduced appetite, may decrease dietary vitamin D intake, while poor diet, and lack of physical activity may also increase the risk of obesity which also has a causal effect on lowering 25(OH)D concentrations [33]. Metabolic demand for vitamin $\mathrm{D}$ may also be increased in those with depression to counter the imbalances in calcium homeostasis associated with this condition [1], which may in turn contribute to $25(\mathrm{OH}) \mathrm{D}$ deficiency. In support of related influences on the association between depression and $25(\mathrm{OH}) \mathrm{D}$, adjustments for basic, socioeconomic and lifestyle factors more than halved the association in our observational analyses.

Our findings may have some clinical implications. For example, depression has been associated with low bone mineral density [34] and an increased risk of osteoarthritis [35]. It is plausible that this adverse association between depression and poor bone health may result from low 25(OH)D concentrations since low 25(OH)D can trigger a compensatory rise in levels of parathyroid hormone (PTH) [36], which not only promotes the conversion of 25(OH)D to its active form, calcitriol, but also increases bone resorption [37]. Indeed, we have recently reported a causal effect of depression on risk of osteoarthritis using the MR approach, highlighting the need to monitor bone health in people with depression [36]. Individuals with depression are also more vulnerable to respiratory infections [38], and this risk may be modifiable by regulating vitamin D levels; lower 25(OH)D concentrations have been causally linked to greater risk of bacterial pneumonias [39], and calcitriol plays a role in modulating the immune response to respiratory viruses [40]. Furthermore, RCTs indicate that the risk of acute respiratory infections can be reduced by daily or weekly vitamin D supplementation [41]. The strengths of this study include the large population size and the breadth of individual data available for participants in the UK Biobank cohort. The use of the MR approach has allowed us to assess bi-directional causality between $25(\mathrm{OH}) \mathrm{D}$ and depression. Genetic markers of 25(OH)D that have arisen from genome-wide association studies are useful instruments for MR analyses of 25(OH)D [42] and we have previously utilized this method to assess the causal relationships between vitamin D status and obesity [33], blood pressure [43], and cognitive function [44]. Similarly, we have used genetically instrumented depression scores to investigate causal effects of depression on BMI [18], and multiple disease outcomes [35]. Although RCT is the gold standard for testing of causality, the MR will help us to avoid methodological problems related to confounding and reverse causality affecting other types of observational studies. Furthermore, this approach allowed us to test the causal link between lifetime nutritional vitamin D status and depression, which most intervention studies struggle to achieve, and which is sometimes 
mentioned as one possible reason for mixed results from RCT [45]. Mixed results from RCTs could also be due to methodological flaws such as lack of baseline $25(\mathrm{OH}) \mathrm{D}$ measures (preventing the ability to demonstrate a change in concentrations), interventions that result in no change in $25(\mathrm{OH}) \mathrm{D}$ concentrations, or reduced rather than increased concentrations, and starting with baseline 25(OH)D levels that indicated sufficiency (not deficiency) [7]. These factors were noted as potential methodological flaws with earlier studies, with evidence for differential conclusions in higher and lower quality studies [7].

There are further methodological considerations with our approach. While we did not detect evidence for a causal role of $25(\mathrm{OH}) \mathrm{D}$ in depression, we cannot rule out the possibility that lower $25(\mathrm{OH}) \mathrm{D}$ makes a small contribution to depression risk. In the combined UK Biobank consortia meta-analyses $(n=424,967)$, our study was powered to detect the OR of 0.92 per $50 \%$ higher serum $25(\mathrm{OH}) \mathrm{D}$, which is a slightly stronger association than that suggested by the observational data $(0.95,0.94$ to 0.96$)$ or MR analyses $(0.97,0.93$ to 1.02$)$. Furthermore, while our results argue against linear increases in $25(\mathrm{OH}) \mathrm{D}$ having a substantial influence on depression, we may not have been able to detect effects of 25(OH)D 'deficiency' with this approach since the instrument captures differences in average $25(\mathrm{OH}) \mathrm{D}$ across the continuum and assumes linear effects. However, given complex biology and the heterogeneity across the different types of 'depression', further studies are warranted to establish more specific associations between $25(\mathrm{OH}) \mathrm{D}$ and, for example, seasonal affective disorder. Although we included self-report and hospital inpatient information, the definition of depression was not gold standard due to lack of a valid depression diagnostic instruments in the UK Biobank [46]. With only $5 \%$ participation rate and some selection towards relatively healthy participants [47], results from the UK Biobank may be prone to collider bias if participation to the study is affected by serum $25(\mathrm{OH}) \mathrm{D}$ and depression status. However, collider bias is unlikely to have had a substantial effect on findings as the estimates were remarkably consistent also when using summary data from consortia that did not include the UK Biobank. As the current study is restricted to white British participants, the findings of this study may not be representative of other populations. Finally, despite efforts to include various MR methods that rely on different pleiotropic assumptions, we cannot fully rule out bias in the causal estimates due to horizontal pleiotropic effects (a situation where the genetic instruments associate with the outcome through pathways other than through the exposure).

In conclusion, we provided genetic evidence that depression contributes to low $25(\mathrm{OH}) \mathrm{D}$ concentrations but found little evidence for a material contribution by vitamin D status on the risk of depression. Our study suggests that while vitamin D may not help to prevent depression, monitoring and treatment of vitamin D deficiency may be beneficial in alleviating adverse influences of depression on health.

Supplementary Materials: The following are available online at https:/ /www.mdpi.com/2072-6 $643 / 13 / 1 / 109 / s 1$. Supplementary note. Mendelian randomization. Table S1. List of 25(OH)Drelated variants, and its association with serum 25(OH)D level among UK Biobank and discovery cohort. Table S2. List of major depressive disorder-related variants used to construct the genetic risk scores. Table S3. Prevalence of depression and summary of $25(\mathrm{OH}) \mathrm{D}$ across different characteristics. Table S4. Association between serum $25(\mathrm{OH}) \mathrm{D}$ level and depression among men and women. Table S5. Association between serum 25(OH)D and depression excluding serum 25(OH)D data from aliquot three blood sample. Table S6. The causal estimates for the association between $25(\mathrm{OH}) \mathrm{D}$ and depression using two, four and all $25(\mathrm{OH}) \mathrm{D}$ variants as the instrument in the two-sample MR analysis. Figure S1. Flow of UK Biobank participants included in the bi-directional analysis between serum 25(OH)D and depression. Figure S2. Association between MDD-related variants and depression in UK Biobank versus discovery GWAS. Figure S3. Comparison of randomized control trial (RCT) with Mendelian randomization (MR) [16] [Panel A], and summary of analyses strategy [Panel B]. Figure S4. Genetic instrument validation. Plot A shows the distribution of $25(\mathrm{OH}) \mathrm{D}$ genetic risk score (GRS), and its association with 25(OH)D in UK Biobank, with the weighted GRS explains $2.7 \%$ of the variability in 25(OH)D. Plot B shows the association between GRS in ten-quantiles and depression in UK Biobank, with the weighted GRS explains $0.2 \%$ of the variability in the depression. Figure S5. 
Plots from two-sample MR analysis of 25(OH)D on depression. Plot A shows the Scatter plot of the estimates of variant-depression association against estimates of variant-25(OH)D association. Plot B shows the funnel plots of instrument strength against causal estimate ( $\beta$ IV). Plot $C$ includes leave-one-out analyses, demonstrating the effect on the overall MR IVW estimate by excluding each of the six variants one at a time. Figure S6. Observational and MR analyses on the association between 25(OH)D (using the 122 25(OH)D-related variants from Revez et al. [26]) and the odds of depression. Figure S7. Plots from two-sample MR analysis of depression on $25(\mathrm{OH}) \mathrm{D}$. Plot A shows the scatter plot of the estimates of variant-25(OH)D association against estimates of variant-depression association. Plot B shows the funnel plots of instrument strength against causal estimate ( $\beta$ IV). Plot $\mathrm{C}$ includes leave-one-out analyses, demonstrating the effect on the overall MR IVW estimate by excluding each of the 44 variants one at a time. Figure S8. Two-sample MR estimates from different MR approaches using 17 major depression-related genetic variants from Hyde et al. [29]. MR Egger intercept $p$-value $=0.87$. Figure S9. Percent change in serum $25(\mathrm{OH}) \mathrm{D}$ associated with depression (Observational), or genetic liability to depression (MR) using 17 major depression-related genetic variants from Hyde et al. [29].

Author Contributions: E.H. supervised the study and advised the analysis; A.M.: Data preparation and analysis; A.M. and A.L. wrote the first draft; all authors interpreted the result, revised the contents. All authors have read and agreed to the published version of the manuscript.

Funding: This research was funded by National Health and Medical Research Council of Australia (GNT1157281).

Institutional Review Board Statement: Ethical approval was granted by the National Information Governance Board for Health and Social Care and North West Multicentre Research Ethics Committee $(11 / \mathrm{NW} / 0382)$, and the study was conducted according to the guideline of the Declaration of Helsinki. The current study is approved by the UK Biobank under application number 10171.

Informed Consent Statement: UK Biobank obtained informed consent from each participant involved in the study.

Data Availability Statement: All data is available through the UK Biobank repository on application.

Conflicts of Interest: All authors declare no conflict of interest.

\section{References}

1. Berridge, M.J. Vitamin D and Depression: Cellular and Regulatory Mechanisms. Pharmacol. Rev. 2017, 69, 80-92. [CrossRef] [PubMed]

2. Menon, V.; Kar, S.K.; Suthar, N.; Nebhinani, N. Vitamin D and Depression: A Critical Appraisal of the Evidence and Future Directions. Indian J. Psychol. Med. 2020, 42, 11-21. [CrossRef] [PubMed]

3. Harms, L.; Burne, T.H.; Eyles, D.W.; McGrath, J.J. Vitamin D and the brain. Best Pract. Res. Clin. Endocrinol. Metab. 2011, 25, 657-669. [CrossRef] [PubMed]

4. Puchacz, E.; Stumpf, W.E.; Stachowiak, E.K.; Stachowiak, M.K. Vitamin D increases expression of the tyrosine hydroxylase gene in adrenal medullary cells. Mol. Brain Res. 1996, 36, 193-196. [CrossRef]

5. Patrick, R.P.; Ames, B.N. Vitamin D hormone regulates serotonin synthesis. Part 1: Relevance for autism. FASEB J. 2014, 28, 2398-2413. [CrossRef] [PubMed]

6. Okereke, O.I.; Reynolds, C.F.; Mischoulon, D.; Chang, G.; Vyas, C.M.; Cook, N.R.; Weinberg, A.; Bubes, V.; Copeland, T.; Friedenberg, G.; et al. Effect of Long-term Vitamin D3 Supplementation vs Placebo on Risk of Depression or Clinically Relevant Depressive Symptoms and on Change in Mood Scores: A Randomized Clinical Trial. JAMA 2020, 324, 471-480. [CrossRef] [PubMed]

7. Gowda, U.; Mutowo, M.P.; Smith, B.J.; Wluka, A.E.; Renzaho, A. Vitamin D supplementation to reduce depression in adults: Meta-analysis of randomized controlled trials. Nutrition 2015, 31, 421-429. [CrossRef]

8. Spedding, S. Vitamin D and Depression: A Systematic Review and Meta-Analysis Comparing Studies with and without Biological Flaws. Nutrients 2014, 6, 1501-1518. [CrossRef]

9. Sarris, J.; Murphy, J.A.; Mischoulon, D.; Papakostas, G.I.; Fava, M.; Berk, M.; Ng, C.H. Adjunctive Nutraceuticals for Depression: A Systematic Review and Meta-Analyses. Am. J. Psychiatry 2016, 173, 575-587. [CrossRef]

10. Jamilian, H.; Amirani, E.; Milajerdi, A.; Kolahdooz, F.; Mirzaei, H.; Zaroudi, M.; Ghaderi, A.; Asemi, Z. The effects of vitamin D supplementation on mental health, and biomarkers of inflammation and oxidative stress in patients with psychiatric disorders: A systematic review and meta-analysis of randomized controlled trials. Prog. Neuro Psychopharmacol. Biol. Psychiatry 2019, 94, 109651. [CrossRef]

11. Tønnesen, R.; Hovind, P.H.; Jensen, L.T.; Schwarz, P. Determinants of vitamin D status in young adults: Influence of lifestyle, sociodemographic and anthropometric factors. BMC Public Health 2016, 16, 385. [CrossRef] [PubMed]

12. Milaneschi, Y.; Peyrot, W.J.; Nivard, M.G.; Mbarek, H.; Boomsma, D.I.; Penninx, B.W. A role for vitamin D and omega-3 fatty acids in major depression? An exploration using genomics. Transl. Psychiatry 2019, 9, 219. [CrossRef] [PubMed] 
13. Michaëlsson, K.; Melhus, H.; Larsson, S.C. Serum 25-Hydroxyvitamin D Concentrations and Major Depression: A Mendelian Randomization Study. Nutrients 2018, 10, 1987. [CrossRef] [PubMed]

14. Libuda, L.; Laabs, B.H.; Ludwig, C.; Bühlmeier, J.; Antel, J.; Hinney, A.; Naaresh, R.; Föcker, M.; Hebebrand, J.; König, I.R.; et al. Vitamin D and the Risk of Depression: A Causal Relationship? Findings from a Mendelian Randomization Study. Nutrients 2019, 11, 1085. [CrossRef] [PubMed]

15. Davies, N.M.; Holmes, M.V.; Smith, G.D. Reading Mendelian randomisation studies: A guide, glossary, and checklist for clinicians BMJ 2018, 362, k601. [CrossRef]

16. Sudlow, C.; Gallacher, J.; Allen, N.; Beral, V.; Burton, P.; Danesh, J.; Downey, P.; Elliott, P.; Green, J.; Landray, M.; et al. UK Biobank: An Open Access Resource for Identifying the Causes of a Wide Range of Complex Diseases of Middle and Old Age. PLoS Med. 2015, 12, e1001779. [CrossRef]

17. Bycroft, C.; Freeman, C.; Petkova, D.; Band, G.; Elliott, L.T.; Sharp, K.; Motyer, A.; Vukcevic, D.; Delaneau, O.; O'Connell, J.; et al. The UK Biobank resource with deep phenotyping and genomic data. Nature 2018, 562, 203-209. [CrossRef]

18. Tyrrell, J.; Mulugeta, A.; Wood, A.R.; Zhou, A.; Beaumont, R.N.; Tuke, M.A.; Jones, S.E.; Ruth, K.S.; Yaghootkar, H.; Sharp, S.; et al. Using genetics to understand the causal influence of higher BMI on depression. Int. J. Epidemiol. 2019, 48, 834-848. [CrossRef]

19. Jiang, X.; O’Reilly, P.F.; Aschard, H.; Hsu, Y.-H.; Richards, J.B.; Dupuis, J.; Ingelsson, E.; Karasik, D.; Pilz, S.; Berry, D.; et al. Genome-wide association study in 79,366 European-ancestry individuals informs the genetic architecture of 25-hydroxyvitamin D levels. Nat. Commun. 2018, 9, 260. [CrossRef]

20. Biobank. Available online: http:/ / www.ukbiobank.ac.uk/ (accessed on 10 October 2017).

21. UK Biobank. Biomark Assay Quality Procedures: Approaches Used to Minimise Systematic and Random Errors (and the Wider Epidemiological Implications). Available online: http://biobank.ctsu.ox.ac.uk/showcase/showcase/docs/biomarker_issues.pdf (accessed on 10 April 2019).

22. Fry, D.; Almond, R.; Moffat, S.; Gordon, M.; Singh, P. UK Biobank Biomarker Project: Companion Document to Accompany Serum Biomarker Data. 2019. Available online: https://biobank.ndph.ox.ac.uk/showcase/showcase/docs/serum_biochemistry.pdf (accessed on 21 May 2020).

23. Jameson, J.L.; Longo, D.L. Precision Medicine-Personalized, Problematic, and Promising. N. Engl. J. Med. 2015, 372, 2229-2234. [CrossRef]

24. WHO. Body Mass Index-BMI. Available online: https://www.euro.who.int/en/health-topics/disease-prevention/nutrition/ahealthy-lifestyle/body-mass-index-bmi (accessed on 16 December 2020).

25. Revez, J.A.; Lin, T.; Qiao, Z.; Xue, A.; Holtz, Y.; Zhu, Z.; Zeng, J.; Wang, H.; Sidorenko, J.; Kemper, K.E.; et al. Genome-wide association study identifies 143 loci associated with 25 hydroxyvitamin D concentration. Nat. Commun. 2020, 11, 1647. [CrossRef]

26. Wray, N.R.; Ripke, S.; Mattheisen, M.; Trzaskowski, M.; Byrne, E.M.; Abdellaoui, A.; Adams, M.J.; Agerbo, E.; Air, T.M.; Andlauer, T.M.F.; et al. Genome-wide association analyses identify 44 risk variants and refine the genetic architecture of major depression. Nat. Genet. 2018, 50, 668-681. [CrossRef] [PubMed]

27. Burgess, S.; Davies, N.M.; Thompson, S.G. Bias due to participant overlap in two-sample Mendelian randomization. Genet. Epidemiol. 2016, 40, 597-608. [CrossRef] [PubMed]

28. Hyde, C.L.; Nagle, M.W.; Tian, C.; Chen, X.; Paciga, S.A.; Wendland, J.R.; Tung, J.Y.; Hinds, D.; Perlis, R.H.; Winslow, A.R. Identification of 15 genetic loci associated with risk of major depression in individuals of European descent. Nat. Genet. 2016, 48, 1031-1036. [CrossRef] [PubMed]

29. Bowden, J.; Davey Smith, G.; Haycock, P.C.; Burgess, S. Consistent Estimation in Mendelian Randomization with Some Invalid Instruments Using a Weighted Median Estimator. Genet. Epidemiol. 2016, 40, 304-314. [CrossRef] [PubMed]

30. Hartwig, F.P.; Smith, G.D.; Bowden, J. Robust inference in summary data Mendelian randomization via the zero modal pleiotropy assumption. Int. J. Epidemiol. 2017, 46, 1985-1998. [CrossRef] [PubMed]

31. Bowden, J.; Davey Smith, G.; Burgess, S. Mendelian randomization with invalid instruments: Effect estimation and bias detection through Egger regression. Int. J. Epidemiol. 2015, 44, 512-525. [CrossRef]

32. Verbanck, M.; Chen, C.Y.; Neale, B.M.; Do, R. Detection of widespread horizontal pleiotropy in causal relationships inferred from Mendelian randomization between complex traits and diseases. Nat. Genet. 2018, 50, 693-698. [CrossRef]

33. Vimaleswaran, K.S.; Berry, D.J.; Lu, C.; Tikkanen, E.; Pilz, S.; Hiraki, L.T.; Cooper, J.D.; Dastani, Z.; Li, R.; Houston, D.K.; et al. Causal Relationship between Obesity and Vitamin D Status: Bi-Directional Mendelian Randomization Analysis of Multiple Cohorts. PLoS Med. 2013, 10, e1001383. [CrossRef]

34. Cizza, G.; Primma, S.; Csako, G. Depression as a risk factor for osteoporosis. Trends Endocrinol. Metab. 2009, 20, 367-373. [CrossRef]

35. Mulugeta, A.; Zhou, A.; King, C.; Hyppönen, E. Association between major depressive disorder and multiple disease outcomes: A phenome-wide Mendelian randomisation study in the UK Biobank. Mol. Psychiatry 2020, 25, 1469-1476. [CrossRef] [PubMed]

36. Oh, R.C. Vitamin D insufficiency as a cause of hyperparathyroidism. Am. Fam. Physician 2005, 71, 46-49. [PubMed]

37. Rejnmark, L.; Ejlsmark-Svensson, H. Effects of PTH and PTH Hypersecretion on Bone: A Clinical Perspective. Curr. Osteoporos. Rep. 2020, 18, 103-114. [CrossRef]

38. Seminog, O.O.; Goldacre, M. Risk of pneumonia and pneumococcal disease in people with severe mental illness: English record linkage studies. Thorax 2013, 68, 171-176. [CrossRef] 
39. Çolak, Y.; Nordestgaard, B.G.; Afzal, S. Low vitamin D and risk of bacterial pneumonias: Mendelian randomisation studies in two population-based cohorts. Thorax 2020. [CrossRef]

40. Greiller, C.L.; Martineau, A.R. Modulation of the Immune Response to Respiratory Viruses by Vitamin D. Nutrients 2015, 7, 4240-4270. [CrossRef]

41. Martineau, A.R.; Jolliffe, D.A.; Greenberg, L.; Aloia, J.F.; Bergman, P.; Dubnov-Raz, G.; Esposito, S.; Ganmaa, D.; Ginde, A.A.; Goodall, E.C.; et al. Vitamin D supplementation to prevent acute respiratory infections: Individual participant data meta-analysis. Health Technol. Assess. 2019, 23, 1-44. [CrossRef] [PubMed]

42. Berry, D.J.; Vimaleswaran, K.S.; Whittaker, J.C.; Hingorani, A.D.; Hyppönen, E. Evaluation of Genetic Markers as Instruments for Mendelian Randomization Studies on Vitamin D. PLoS ONE 2012, 7, e37465. [CrossRef] [PubMed]

43. Vimaleswaran, K.S.; Cavadino, A.; Berry, D.J.; Jorde, R.; Dieffenbach, A.K.; Lu, C.; Alves, A.C.; Heerspink, H.J.L.; Tikkanen, E.; Eriksson, J.; et al. Association of vitamin D status with arterial blood pressure and hypertension risk: A mendelian randomisation study. Lancet Diabetes Endocrinol. 2014, 2, 719-729. [CrossRef]

44. Maddock, J.; Zhou, A.; Cavadino, A.; Kuźma, E.; Bao, Y.; Smart, M.C.; Saum, K.U.; Schöttker, B.; Engmann, J.; Kjærgaard, M.; et al. Vitamin D and cognitive function: A Mendelian randomisation study. Sci. Rep. 2017, 7, 13230. [CrossRef]

45. Scragg, R. Limitations of vitamin D supplementation trials: Why observational studies will continue to help determine the role of vitamin D in Health. J. Steroid Biochem. Mol. Biol. 2018, 177, 6-9. [CrossRef] [PubMed]

46. Smith, D.J.; Nicholl, B.I.; Cullen, B.; Martin, D.; Ul-Haq, Z.; Evans, J.; Gill, J.M.R.; Roberts, B.; Gallacher, J.; Mackay, D.; et al. Prevalence and Characteristics of Probable Major Depression and Bipolar Disorder within UK Biobank: Cross-Sectional Study of 172,751 Participants. PLoS ONE 2013, 8, e75362. [CrossRef] [PubMed]

47. Fry, A.; Littlejohns, T.J.; Sudlow, C.; Doherty, N.; Adamska, L.; Sprosen, T.; Collins, R.; Allen, N.E. Comparison of Sociodemographic and Health-Related Characteristics of UK Biobank Participants With Those of the General Population. Am. J. Epidemiol. 2017, 186, 1026-1034. [CrossRef] [PubMed] 